\title{
Detection of auditory signals presented at random times, II
}

\author{
R. DUNCAN LUCE, INSTITUTE FOR ADVANCED STUDY, PRINCETON, NEW JERSEY ${ }^{2}$ \\ AND DAVID M. GREEN ${ }^{3}$ UNIVERSITY OF CALIFORNIA, SAN DIEGO
}

The paper includes experimental results obtained using two procedures in which signals are presented at random times. $A$ simple three-stage theory consisting of a sensory process, followed by a response bias, and ending in a response process, involving a random delay, and that may or may not have a memory, is compared with the data. The free-response procedure yielded data that could not be accounted for by a first version of the theory in which the crucial assumption about the response process was that once a response process is under way it locks out all further inputs until the response is made. Alternative models, including a race between response processes with the first response suppressing all others and a simple first-in first-out queue, were equally inadequate. On the assumption that the difficulties lie in the complexities of the response process when there are two or more inputs close in time, we decided to avoid these theoretical difficulties by using a modified reaction-time procedure. The initial results are encouraging. The tails of the latency distributions appear to be exponentially distributed and a theoretical prediction that two of the time constants should be the same appears to be supported. The major indications of difficulties are inconclusive evidence that the response bias parameter may not be constant and, possibly related, that the initial portion of the tails may overshoot the predicted value. In spite of the possible variation in the bias parameter, estimates of the signal-plus-noise to noise-alone parameters increase in a systematic manner with signal strength.

Virtually all sensory discrimination experiments use clearly specified intervals of time during which a signal may or may not be presented. The $O$ need not attend at other times since signals are presented only during these specific intervals. A response is required shortly af ter each interval or each group of intervals, and the $O$ 's performance is summarized as estimates of certain conditional response probabilities. For example, in the Yes-No design a response occurs after each interval and we estimate the probability of a detection (Yes response) conditional on the signal being presented and also conditional on its not being presented.

Such experiments are as artificial as they are convenient to analyze. Seldom in life do we receive advance warning that a signal may shortly occur, let alone know exactly the interval of time during which to attend. In most realistic situations, signals occur in a highly unpredictable manner, even if they do not occur in a perfectly random manner.

We continue our attempt to develop a rigorous analysis of detection in experiments that try to mimic the temporal uncertainty of real life. A first pass at a theory was given in Luce (1966); it was followed by an experiment and some additional theory in Green and Luce (1967). Here we report a replication and a modified experimental design, both using better equipment, and we consider various modifications of the theory.

\section{GENERAL THEORY}

The contrast between our methods and those used by earlier investigators (for example, Broadbent \& Gregory, 1963; Egan, Greenberg, \& Schulman, 1961; Watson \& Nichols, 1966) is particularly striking because we ignore the responses that occur shortly after other observable events-in particular, those shortly after a signal-and concentrate mainly on the tails of certain latency distributions. Several different distributions arise by considering interresponse times (IRT) and signal-response times (SRT) conditioned on various things being true. ${ }^{4}$ With a Poisson schedule-the continuous analogue of a uniform distribution-of signal presentations, the theory predicts that the tails of these distributions are exponential with time constants that are simple functions of theoretical sensory and response bias parameters. So our attention is focused, first, on whether the tails are in fact exponential and, second, on the numerical values of the exponential time constants. Inferences about the sensory system and the discriminability of signals are made from these estimated parameters.

To see in more detail what is involved, consider the block diagram of Fig. 1, which presents the essential structural components of the theory. The "Sensory Process" box represents the mechanism responsible for detecting faint signals. In the absence of any input signal, the output of this box is generally quiet. Occasionally, however, according to a random (Poisson) schedule, it yields an output, thereby falsely indicating the presence of a signal. We denote the intensity parameter of this "noise" process by $v$. When a signal is actually presented, the tendency for the box to issue an output is momentarily increased by an amount that depends upon the signal strength; after the signal ends, the process returns to its resting Poisson level. In principle, the value of the Poisson parameter $v$ could depend on many things other than the stimulating conditions and the sensory system of the $O$, but we shall assume that it depends only on these. In particular, we assume that it is unaffected by the density of signal occurrences, the values and costs of various responses, and the instructions to the $O$.

The Sensory Process output is fed into the next box, labeled "Response Bias," which simply determines with some constant and statistically independent probability, b, whether or not an alleged detection shall be permitted to initiate a response. We hope that $b$ can be treated as independent of changes in the sensory input and that much of the individual response variation caused by altering instructions and payoffs can be accounted for by changes in $b$. If so, then we can separate analytically the response and the sensory processes. Observe that when no signal is present the output of the Response Bias box is Poisson, but with intensity b $v$ rather than just $v$. One experimental task is to

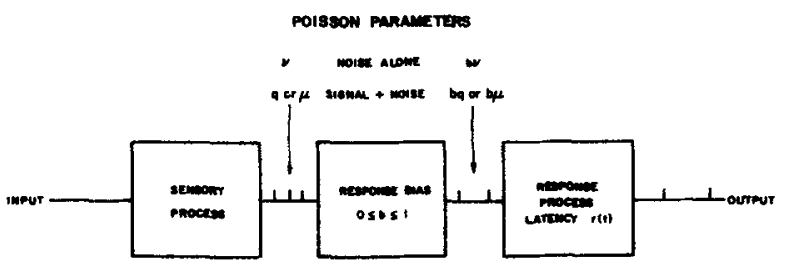

Fig. 1. Functional parts of the general theory. The output events of the Sensory Process and Response Bias are assumed to be Poisson distributed with the indicated parameters when noise or signal-plus-noise are present at the input. 
try to verify that the assumed dependencies and independencies of $b$ and $v$ are valid.

The output of the Response Bias is fed into the third box, labeled "Response Process," which introduces a delay before the observed response occurs. When a reaction is initiated, we assume that before the response occurs there is an unobservable delay, called the reaction latency, that this latency is a bounded random variable, with density $r$, and that it is independent of the random processes in the other two boxes. By a bounded random variable we simply mean that for some $\tau>0, r(t)=0$ for all $t \leqslant 0$ and for all $t \geqslant \tau$.

In addition to these assumptions, we must consider carefully the conditions under which a reaction is, in fact, initiated. The problem is to know what happens when two or more inputs occur so close in time that the response to the first has not yet occurred when the second arrives. Various assumptions about what happens generate different models within the same general theoretical framework. We describe one now and two others later. Unfortunately, they all seem to be wrong.

\section{Lock-Out Model}

A basic question is whether the Response Process box is capable of storing an input until a reaction that is under way produces a response or whether the second input is "locked out" and hence lost. By far the simplest, but almost surely a wrong, assumption is that any input arriving while another one is being processed is lost. Such a system has no memory, and so each time a response is made we can begin our analysis at the time the response occurs and all events preceding the response can be ignored. This leads to great mathematical simplicity, especially when the input to the Response Process is Poisson. Another way to view this lock-out model is that whenever the Response Process is busy processing an input, a feedback loop to the Response Bias box reduces the bias from $b$ to 0 ; when the response is made, it is returned to $b$.

Given the lock-out model and a Poisson schedule of stimulus presentations, Green and Luce (1967) worked out four, different, conditional IRT and SRT distributions. For observed times $t$ greater than $\tau$, each distribution is exponential and has as time constant either $b v+\lambda$ or $b v+b q \lambda$, where $\lambda$ is the Poisson parameter of the stimulus presentation schedule and $q$ denotes the probability that the Sensory. Process emits an output when a signal is presented. The stimulus presentation parameter, $\lambda$, is an experimental parameter, so it is known. We can estimate bu from an estimate of $b v+\lambda$, and with that known, we can estimate bq from the estimate of $b v+b q \lambda$. The ratio, $q / v=b q / b v$, is a measure of the signal's detectability not unlike $d^{\prime}$ in the signal detectability analysis of fixed interval detection. Since the response bias, $\mathrm{b}$, cancels in determining the ratio $\mathrm{q} / v$, this measure of the signal's detectability is presumably independent of response bias.

\section{Test of Lock-Out Model}

Previous free-response experiment. In the earlier paper, we reported an analysis of data from an experiment in which signals of short duration and fixed intensity were presented according to a Poisson schedule. The results were equivocal.

A key prediction was that all four distributions have exponential tails. For one $\mathrm{O}$, this was approximately true, but the other $O$ exhibited consistent departures from the exponential. Moreover, to do as well as we did, we were forced to set the upper bound, $\tau$, on reaction latencies at $2 \mathrm{sec}$. Although initially this struck us as excessively long, we ultimately persuaded ourselves that it might have resulted from our inability to motivate the Os to respond rapidly. The equipment used in that experiment did not permit us to analyze the data immediately

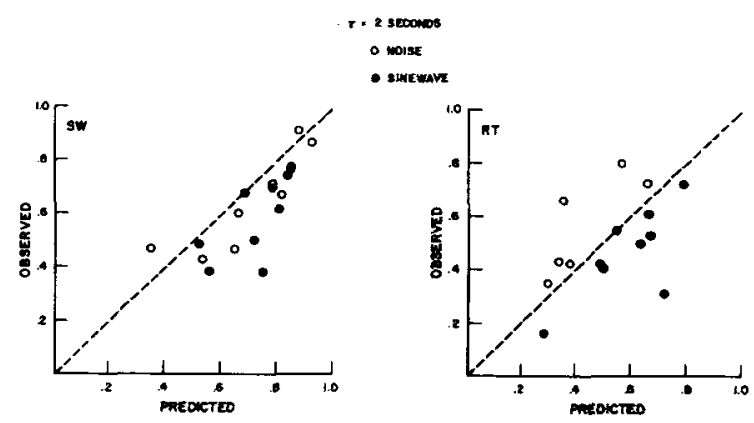

Fig. 2. Corrected scatter diagrams for the free-response experiment of Green and Luce (1967). The figure displays the observed vs predicted proportion of responses occurring within $\tau=2 \mathrm{sec}$ of a signal onset given no observable events within $\tau=2$ sec before the signal. The obtained percentage is the percentage of response falling within this interval. The prediction is based on the lock-out model using parameters estimated from the tails of several distributions (see text).

and therefore we could not assess, much less motivate, rapid responses. Actually, two other discrepancies seemed far more serious.

First, the proportion of pairs of responses less than $200 \mathrm{msec}$ apart was much larger than predicted by the theory. These pairs, we argued, probably arose from a failure of the lock-out assumption. Rather, the data suggested that when a signal arrives after a reaction has been initiated, but before the response is made, the new arrival is stored and is processed immediately af ter the first response. Naturally, slow reaction latencies increased materially the opportunities for this to happen.

Second, the model consistently failed to predict the probability of a response occurring with the $\tau=2$-sec period following the presentation of a signal. A comparison of the predicted and obtained values is shown in Fig. 6 of Green and Luce (1967), but the published graph is incorrect. ${ }^{5}$ The correct scatter diagrams are shown in Fig. 2. As can be seen in the graph, the theory tends to predict a greater proportion of responses falling shortly after the onset of the signal than is obtained experimentally, especially for O SW.

Replication. The present results were obtained in a new laboratory with better equipment, including a PDP-9 digital computer that controlled the experiment and immediately monitored the O's performance. Among other things, it permitted us to provide the Os with immediate feedback.

We first replicated certain conditions of the previous free-response experiment. Despite some minor changes in procedure, the same three discrepancies appeared. So, af ter some preliminary testing, we decided to instruct the Os not to respond to any detections that occurred while a response was in progress. The aim of the change was to determine if the Os could behave in a fashion consistent with the lock-out assumption. This instruction sharply reduced the proportion of pairs of responses close together in time. Nonetheless, we must keep in mind that the lock-out assumption is almost certainly wrong when Os are left uninstructed on this point.

We also introduced a system of immediate feedback that was aimed at encouraging both fast responses and the adoption of the lock-out mode of behavior. The feedback system operated as follows. After each response, the computer determined whether the immediately preceding event was a signal or a response. If a response, one of five lights was lighted. If a signal, the computer measured the time between its onset and the response. The first light was lit if the time between signal onset and response was 


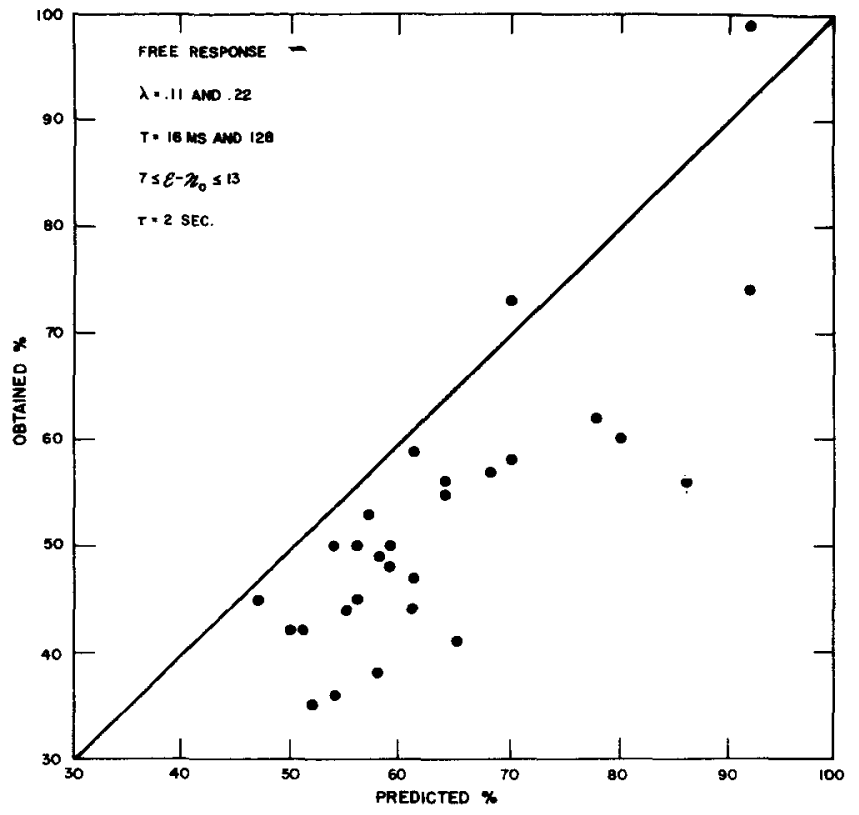

Fig. 3. The same as $F$ ig. 2 for the present free-response experiment with $\tau=2 \mathrm{sec}$. The various experimental parameters used to obtain the points are indicated, $T$ is signal duration, $\&-\eta_{0}$ is the ratio of signal energy to noise power density in decibels and $1 / \lambda$ is the mean wait in seconds between signal occurrences.

$0-300 \mathrm{msec}$, the second if the elapsed time was $300-500 \mathrm{msec}$, the third if $500-1,000 \mathrm{msec}$, and the fourth if elapsed time exceeded 1,000 msec. Positive rewards were given for latencies in the first three categories and a penalty was imposed for responses that either preceded the signal (false alarms) or exceeded $1,000 \mathrm{msec}$. Also, a counter, visible to the $O$, accumulated the winnings or losses during the trial session. Obviously, the penalty for false alarms supplemented materially the lock-out instructions.

Although our experimental control was much improved, sizable discrepancies between the lock-out model and the data remain. The most notable is shown in Fig. 3 in which the obtained and predicted proportion of responses within $\tau=2 \mathrm{sec}$ of a signal are plotted. The failure of the prediction is painfully apparent. Only 2 out of the 28 points fall above the predicted line, and some of those below it are as much as a factor of two below. The various points in Fig. 3 come from a variety of experimental conditions using several signal-to-noise ratios, two signal durations, $16 \mathrm{msec}$ and $128 \mathrm{msec}$, and two signal presentation parameters, $\lambda=0.11 \mathrm{sec}^{-1}$ and $0.22 \mathrm{sec}^{-1}$. Most points represent the data from a daily run that consisted of four $15-\mathrm{min}$ sessions conducted during a $2-\mathrm{h}$ period. The actual number of stimuli presented depended on the signal density, whereas the number of responses depended both on that and on the $O$ 's criterion for making a response. Only those percentages that were estimated from at least 100 responses are shown. Some of the percentages are estimated from as many as 400 responses.

Various hypotheses have been explored in an attempt to pinpoint the factors responsible for the discrepancy. Although no cause can be blamed with certainty, we are convinced by indirect evidence that the basic problem probably lies in the assumptions about the response process. Two sources of evidence are the following. First, those conditions, such as a liberal response criterion or a high density of signals, that lead to a high density of responses exhibited the largest discrepancies between theory and data. Second, a detailed chi-square analysis was made of the IRTs conditional on no intermediate signal to see just how well the exponential distribution fitted the data. It was found that when, in addition to no intermediate signal, we also required that no observable event-either a response or signal-lie in the $\tau=2 \mathrm{sec}$ before the first of the two responses, the chi-square values were decreased approximately $30 \%$ to about chance levels. This strongly suggests that the exponential assumption is not quite correct unless the data are for successive responses that are well separated in time from other events.

Neither piece of evidence is completely persuasive that the lock-out assumption is the problem, because the same data could arise if the sensory system were not homogeneous in time. Although it is probably not exactly homogeneous, it seems rather doubtful that large inhomogeneities should extend over such prolonged periods as 2 sec.

\section{Alternative Models}

In an effort to understand the discrepancy between the lock-out model and the data, we investigated three modifications of the theory. Two of them altered the basic assumptions about the response process, and the third treated the signal as finite in duration rather than as the idealized point assumed in the initial theory. We sketch the details of each model in the appendices, but since all the modifications are as inadequate as the original lock-out model in predicting the results shown in Figs. 2 and 3, we do not devote much space to them here.

All of the models of the free-response experiment predict the various IRT and SRT distributions. In the appendices, we derive for each model the expected density, $f_{i}$, for each of the following observable times.

\section{Description of Density}

$f_{1}(t)$ Unconditional interresponse times.

$f_{2}(t)$ Interresponse times conditional on no signal between the two responses.

$f_{3}(t)$ The times from a signal until the next response conditional on no observable event (either a signal or response) during the $\tau \sec$ prior to the signal. (Note: Other signals may occur between signal and response.)

$f_{4}(t)$ The times $t$ from a signal until the next response conditional on no observable event (either a signal or response) during the $\tau$ sec prior to the given signal and on no observable event (a signal) from the signal until the response.

$f_{5}(t)$ The times from a signal to a response conditional on no other signal between the previous response and the given signal.

The first four densities are the same as those studied in Green and Luce (1967). The fifth density is useful since the various models predict slightly different forms for it. We turn now to the first of our alternate models.

Response suppression model. In the response suppression model, each input to the Response Process is assumed to initiate a reaction. When two or more reactions are under way, they are carried out independently and in parallel, as in a race, until a response occurs. At that point, all uncompleted reactions are terminated without any other response occurring and the whole process begins anew. This model, like the lock-out model, has the property that there is no memory for events prior to the previous response. 
In Appendix 1, equations are derived for the five IRT and SRT distributions of this model. For $t \geqslant \tau$, all of these distributions are exponential with time constants $b \eta=b v+\lambda b q$ for 1,3 , and 5 and bv $+\lambda$ for 2 and 4 (Eqs. $7,10,13,16,17$ ). This is exactly the same as for the lock-out model. Although the expressions for

$$
\int_{0}^{\tau} f_{i}(t) d t
$$

differ from those of the lock-out model, the crucial one is similar in form, namely,

$$
\int_{0}^{\tau} f_{4}(t) d t=1-\left[\frac{1-b q}{1+\lambda \frac{b q}{b v} R_{-(b v+\lambda)}}\right] e^{-(b v+\lambda) \tau},
$$

where

$$
R_{\theta}=\int_{0}^{\infty} e^{\theta t} r(t) d t=\int_{0}^{\tau} e^{\theta t} r(t) d t
$$

In the lock-out model we have $R_{-} \lambda / R_{b v}$ rather than $R_{-}(b v+\lambda)$, but we show that this makes precious little difference.

Observe that for $\theta \geqslant 0$,

$$
1 \leqslant \mathrm{R}_{\theta} \leqslant \mathrm{e}^{\theta \tau} \text { and } \mathrm{e}^{-\theta \tau} \leqslant \mathrm{R}_{-} \boldsymbol{\theta} \leqslant 1
$$

Thus,

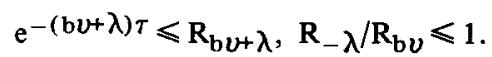

Using these upper and lower bounds, we see that the possible range in values for

$$
\int_{0}^{\tau} f_{4}(t) d t
$$

for the two models is

$$
\begin{aligned}
\mathrm{e}^{-(\mathrm{b} v+\lambda) \tau(1-b q)}\left(\frac{1}{\left.1+\frac{\lambda b q}{b v} \mathrm{e}^{-(\mathrm{b} v+\lambda) \tau}-\frac{1}{1+\frac{\lambda b q}{\mathrm{~b} v}}\right)}\right. \\
=\frac{(1-\mathrm{bq}) \mathrm{bq} \mathrm{e}^{-(\mathrm{b} v+\lambda) \tau}\left[1-\mathrm{e}^{-(\mathrm{b} v+\lambda) \tau] \frac{\lambda}{\mathrm{b} v}}\right.}{\left[1+\frac{\lambda \mathrm{bq}}{\mathrm{b} v} \mathrm{e}^{-(\mathrm{b} v+\lambda) \tau]\left[1+\frac{\lambda \mathrm{bq}}{\mathrm{b} v}\right]}\right.} \\
\leqslant(1-\mathrm{bq}) \mathrm{bq} \mathrm{e}^{-(\mathrm{b} v+\lambda) \tau}\left[1-\mathrm{e}^{-(\mathrm{b} v+\lambda) \tau] \frac{\lambda}{\mathrm{b} v}}\right.
\end{aligned}
$$

Terms of the form $(1-p) p$, where $0 \leqslant p \leqslant 1$, have a maximum value of $1 / 4$, and so the range is bounded by

$$
\frac{1}{16} \frac{\lambda}{b v}
$$

In our data, $\lambda \leqslant b v$, so the range is no greater than $1 / 16 \approx 0.06$ (in many cases, it is a good deal less since we have been rather generous in our inequalities). It is clear that a shift of 0.06 is inadequate to cure Fig. 3.

Simple queue model. A model with, perhaps, the simplest form of memory can be found in the literature on queues under the title "the single-server queue with random arrivals and a general distribution of service-times [Cox \& Smith, 1961, pp. 50-58]." It assumes, as do we, a Poisson schedule of arrivals and an arbitrary density of reaction latencies (i.e., server times). The memory or storage system is of the "first-in-first-out" type: an input immediately initiates a reaction if none is already in progress; otherwise, the input joins the queue and initiates a reaction only after each of the preceding inputs has led to a response.

Let $b \eta=b v+\lambda b q$ be the Poisson parameter associated with the combination of noise and signal input with signal density, $\lambda$. Denote the mean reaction latency by $\overrightarrow{\mathrm{r}}$, i.e.,

$$
\bar{r}=\int_{0}^{\infty} t r(t) d t=\int_{0}^{\tau} t r(t) d t
$$

Then, provided that $\rho=b \eta \overline{\bar{r}}<1$, it is known that $\rho$ is the asymptotic probability that when a response is made there is at least one input in storage waiting to initiate a reaction. Since we are confident that $\bar{\tau} \leqslant 1$ and estimates of $b \eta$ are $<1$, the condition $\rho<1$ is met. Equations for $\mathrm{f}_{\mathrm{i}}, \mathrm{i}=1, \cdots, 5$, are derived in Appendix 2 (eqs. 18-22). Again, the tails are exactly as in the lock-out and response suppression models. In addition, the equation for

$$
\int_{0}^{\tau} f_{4}(t) d t
$$

is identical to that of the lock-out model. It is true that the equations to estimate $R_{-} \lambda$ and $R_{b v}$ are different, but as the argument just given shows, these changes cannot possibly be large enough to affect the predictions significantly.

Finite signal duration model. We began to wonder if our difficulties could possibly stem from the idealization of a signal as a point in time when, in fact, the signal duration was, initially, about 1/10 sec. So we developed (see Appendix 3) the lock-out model on the assumption that each signal could be represented as a Poisson process with parameter b $\mu$ and duration $\delta$, where $\delta$ is so small (on the order of $0.1 \mathrm{sec}$ ) that $\delta^{2}$ and higher powers are negligible.

Since $\tau$ is at least an order of magnitude larger than $\delta$, this change is of small matter. The equations of the finite signal duration model which are given in Appendix 3, are somewhat different in form from those derived in the original lock-out model. However, because $\lambda, \delta$, and bv are all in the neighborhood of 0.1 , the equations are, to an excellent first approximation, the same as those given in the original lock-out model.

Some of the data displayed in Fig. 3 are based on a duration of $16 \mathrm{msec}$ rather than $128 \mathrm{msec}$, in which case the approximations of the finite duration model are even closer to the original lock-out equation. The data taken at the shorter duration are about as discrepant from the theory as those data taken at the longer duration.

Before closing our discussion of alternative models, we should mention two others we have thought about, although as yet we nave not managed to derive the latency distributions. The first is the simple parallel processor, or queue with an infinite number of identical servers, in which every input to the Response Process initiates a reaction that always terminates in a response, and when two or more reactions are under way they proceed independently. The second model-really a class of models-assumes that the basic difficulty lies not in the assumptions about the Response Process, but in the assumption that the response bias is constant. Later data (Figs. 6 and 7) suggest that possibly the tails of some distributions may fall off faster than exponential, which, if true, probably means that the $O$ becomes impatient with very long interresponse times. Thus, 
instead of assuming constant parameters, bv and bq, we might assume simple proportional increases of the form t $v$ and tq, where $t$ is the time since the last response.

\section{Reaction-Time Model}

Our failure to arrive at a model to explain the free-response data led us to try to subdivide the difficulties. Could we devise an experiment that would permit us to study the sensory and response bias processes without becoming embroiled in the complexities of interacting response processes?

Since the interaction of more than one response process appeared to be the main source of our difficulties, we wanted a procedure in which the $\mathbf{O}$ emitted one, and only one, response. One way to accomplish this is simply to terminate the experiment for some period of time following each response. No signals occur and the $\mathrm{O}$ is instructed not to respond during this interval. In effect, this changes the free-response situation to a simple reaction-time experiment. There are, however, three rather unusual features of such a reaction-time experiment. First, the signals are difficult to detect, as in most detection experiments. Second, the time from the beginning of each trial to the occurrence of the onset of the signal is exponentially distributed (random fore-period); this occurs because it is the distribution of waiting times from a response to the next signal in the free-response experiment. Third, the signal remains on until a response occurs. Under these conditions and with a strong motivation for fast responses, many responses occurred before the signal arrived. We therefore did not need to introduce "catch trials" to estimate the tendency for a false alarm, and, since a response always occurred within a few seconds after the signal onset, we were not forced artificially to terminate a trial because no response occurred.

Since the signal stays on until the response occurs, we assume that the signal merely increases the Poisson parameter bv, associated with noise, to a larger value $b \mu$, associated with signal plus noise. (We also worked out the theory for the case of discrete signals, but the results are simpler in this case.) The theory permits us to derive (see Appendix 4) expressions for the distributions of the three independent distributions illustrated in Fig. 4. On those trials when the response precedes the signal, we simply measure the time to the response. The distribution of these times is called the false-alarm density and is denoted $f_{R}$. On those trials when the signal comes first, we measure both the time to the signal and the time from the signal to the response. The distribution of the former times is called the signal-wait density, $f_{S}$, and of the latter times, the reaction-time density, $f_{R-S}$ Because the signal-wait density is conditional on the signal preceding the response, $f_{S}(t)$ is not the same as the signal presentation density, $\lambda e^{-\lambda t}$.

The theory predicts that the tails $(t \geqslant \tau)$ of each of these distributions is exponential with parameters $b v+\lambda$, $b v+\lambda$, and b $\mu$ (Eqs. 35, 40, 37, respectively). So we first check for deviations from the exponential prediction. If that hypothesis is sustained, we then test whether or not the time constants from the tails of the $f_{R}$ and $f_{S}$ distributions are the same. If so, then we estimate $b v$ and $b \mu$ and test whether $b \mu / b v$ is independent of our manipulation of the response criterion. Finally, we examine how $\mathrm{b} \mu / \mathrm{bu}$ varies with signal-to-noise ratio for a fixed response criterion; this function is of special interest if the ratio is independent of $b$.

\section{Reaction-Time Experiment}

Two Os (one male and one female) were run in a sound-treated cubicle. They wore TDH-39 earphones mounted in sound-absorbing circumaural cushions, and throughout the experiment there was a background of continuous noise. Each

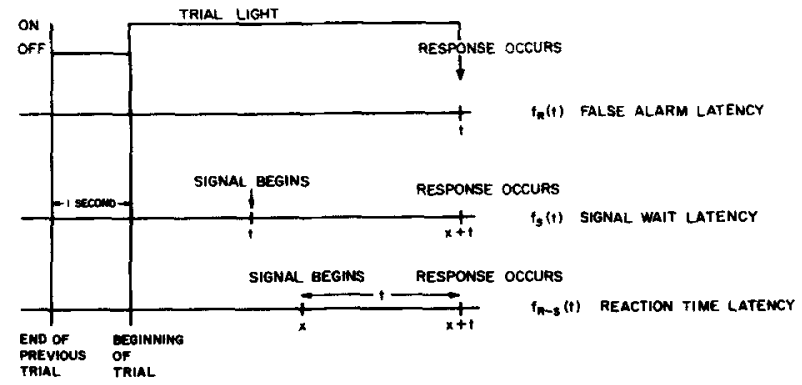

Fig. 4. Schematic representation of the types of trials that can occur in the reaction-time experiment and the three latency measures.

trial began with the onset of a warning light and after a random delay (foreperiod) a $1,000-\mathrm{Hz}$ sinusoid was turned on at a zero crossing. Both the light and the signal remained on until the $O$ responded by pressing a key, at which point both were extinguished and information feedback was provided. When the response preceded the signal, the signal was suppressed, the light was extinguished, and feedback was provided. After a fixed delay of about 1 sec the next trial began.

The random delay was distributed exponentially with a mean waiting time of about $5 \mathrm{sec}$, i.e., $\lambda=0.22 \mathrm{sec}^{-1}$. It is well to recall that the exponential distribution has the property that if the event has failed to occur by time $t$, then its probability of occurring in the interval from $t$ to $t+\Delta$ is independent of $t$. For this reason the exponential distribution, but not the various others generally used in reaction-time experiments, is the appropriate generalization of a uniform distribution on a finite set.

The feedback was provided by lighting one of five lights. This indicated whether or not the response preceded the signal and, if not, how quickly it came after the signal. Payoff in points was associated with these five categories as follows:

\section{Category $<0 \quad 0-300 \mathrm{msec} \quad 300-400 \mathrm{msec} \quad 400-500 \mathrm{msec}>500 \mathrm{msec}$}

$\begin{array}{llllll}\text { Payoff } & -\mathrm{c} & 5 & 3 & 1 & -\mathrm{c}\end{array}$

We manipulated two experimental variables.

Signal-to-noise ratio. The noise was held constant at a spectrum level of about $50 \mathrm{~dB}$ while the signal intensity was varied. The signal duration varied from trial to trial since it depended on how quickly the $O$ responded. Thus we can only specify the signal power, $\mathrm{P}$ (signal energy per second), to noise power density, $\mathbf{N}_{0}$ (noise power per cycle per second), as the basic independent variable. Six signal-to-noise ratios were used in the experiment, ranging from 11.5 to $24.5 \mathrm{~dB}$. The lowest level was such that if this level were used in a two-alternative, forced-choice design, with clearly marked intervals and a signal of $1 / 10$-sec duration, a typical $O$ would perform at the chance level of $50 \%$ correct. For the highest intensity, this percentage would be about $80 \%$. Naturally, it was more difficult to detect a signal in the reaction-time experiment because its onset was uncertain; however, the fact that it was terminated only by the response permitted the $\mathrm{O}$ to increase his effective integration time.

Response criterion. In a second experimental series, we fixed the signal at an intermediate level of difficulty $\left(10 \log P / N_{0}=\right.$ $16.5 \mathrm{~dB}$ ) and asked the $O$ to vary his criterion for initiating a response. In preliminary sessions, we attempted to manipulate his criterion by varying the cost, $-c$, of false alarms and too-slow reactions. As the two Os differed in their sensitivity to changes in $c$ and as large behavioral changes resulted in rather small changes 


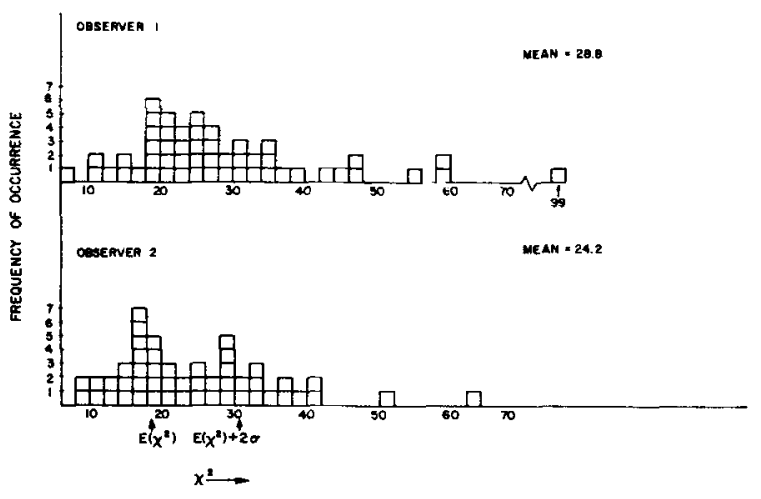

Fig. 5. Histograms of chi square for a maximum likelihood fit of an exponential distribution to the tails of the three latency distributions, $\tau=1 / 2 \mathrm{sec}$, measured in the reaction-time distribution. Each chi-square value is based on at least 100 points, i.e., at least five observations per cell. The expected value of the chisquare statistic and the upper two-sigma values are indicated on the graphs.

in the overall payoff, we decided that it would be better to manipulate the criterion more directly. We monitored the ratio of the number of false alarms to the actual number of stimuli presented and we asked the Os to maintain that ratio in one of three regions: 0.3 to $0.5,1.0$ to 1.5 , or 3.0 to 5.0 . Given that the ratio fell within the prescribed region, we counted the number of points won according to the above schedule, with $\mathrm{c}$ set at unity, and we rewarded the winner with a bonus of $\$ 0.25$ at the end of the run. The two Os competed with each other, and could win as much as an extra dollar for each 2 -h session. They responded to this procedure, were highly motivated, and seemed to enjoy the sessions.

\section{Results}

Form of the tails. To determine how closely the tails of $f_{R}, f_{S}$, and $f_{R-S}$ conform to the exponential prediction of the theory, we proceeded as follows. We divided each experimental condition into first-half and second-half, which, with nine experimental conditions and three distributions, resulted in a total of 54 frequency distributions. Some (e.g., $f_{R}-S$ at the higher signal-to-noise ratios) included too few latencies to make a meaningful comparison possible; we report only those with at least 100 observations. For each frequency distribution, we subtracted $\tau=1 / 2 \mathrm{sec}$ from each time, discarded all negative results, and calculated the reciprocal of the mean of all the positive ones. This gave us the maximum likelihood estimate of the exponential time constant for the tail. We then divided the theoretical distribution into 20 equally likely intervals and compared the obtained and expected numbers in these intervals by the chi-square statistic. Figure 5 shows the resulting distribution of chi square for each $O$. The expected value is 19 and the expected SD is about 6.2. Observer 2's data are somewhat less variable and nearer the expected value than are those of $O 1$. Clearly, the variability is somewhat greater than would be expected by chance alone, but since chi square is a notoriously sensitive statistic, we are reasonably content with the overall pattern.

In an attempt to see if there might be some systematic deviation from the exponential, we averaged the percentages from each of the 20 intervals over the various signal-to-noise ratios and over the three response criteria. The results of these analyses are plotted in Figs. 6 and 7.

Each panel represents a composite of the particular latency distribution, $f_{R}, f_{S}$, or $f_{R-S}$, for each $O$ in the two experiments. The insert in each panel shows the percentage of latencies falling within each of the 20 intervals of the theoretical exponential density used to fit the data. The expected percentage is $5 \%$ and the observed percentage usually falls within a range of $4 \%$ to $6 \%$. These same percentages were used to construct the histograms shown in the main portion of the panels. Here the averaged observed percentages have been scaled to be proportional to an area contained in that interval of the exponential function. Since the time scale is linear, the width of these regions containing equal areas is narrow for short times and wider for long times. The smooth line is, of course, the exponential curve. Despite some possible departures of the data from the expected curve, the exponential function provides an excellent first-order description of the tail of the distribution of times observed in this experiment. Lucas (1967) has also presented data from a different experimental task indicating that a distribution similar to $f_{R}$ has an exponential tail.

Two panels display data that may indicate a systematic departure from the exponential tail. These occur in the reaction-time distributions, $f_{R}-s$, for the experiment in which the signal level was varied (Fig. 6). It appears that more short reaction times are observed than would be expected on the basis of the theory. Our first thought was that we had chosen too small a value for $\tau$, and so were not at a point where $r(t)$ was zero thereby contaminating the beginning of the tail distribution by the end of the reaction latency. Further reflection indicates that this cannot be the explanation. In fact, one can prove, generally, that when too small a value of $\tau$ is used in analyzing these tails, the departure must be in the other direction-that is, the observed percentage should on the average be less than the predicted value.

Whether this effect is genuine is not easy to tell from these data. The consistent departure appears to occur largely when the signal level is high. (Note that the departure is not as evident for either $O$ in the second experiment in which the signal level was at the middle of the range of levels used in the first experiment.) At high signal intensities there is, of course, little data in the tail since most of the responses occur shortly after the onset of the signal. However, because the theory does not appear to have any easy way to account for this discrepancy, we plan to collect data with fairly loud signals and very stringent criteria to investigate this problem in more detail.

Equality of $f_{R}$ and $f_{S}$ tail parameters. According to the theory (Eqs. 35 and $\mathbf{4 0}$ in Appendix 4), the exponential tail parameters of $f_{R}$ and $f_{S}$ should be identical. As a tail parameter is estimated by the reciprocal of the mean of times beyond $\tau=1 / 2$, it does not really matter whether we compare the parameter estimates or the means, but the means are more convenient since their sampling distribution is specified by the theory. The scatter diagram of the two means for the two Os is shown in Fig. 8. Accepting for the moment the $2 \sigma$ ellipse shown on the graph as a reasonable measure of variability, there does not seem to be any strong, systematic departure from the predicted equality, but the scatter does seem excessive. Clearly, a regression line fit to the data of 02 would not lie on the main diagonal, but there simply is not a sufficiently large range in the values of the means to be sure if this trend is real.

The variability associated with each point displayed in Fig. 8 is different. Some of the data points are based on thousands of observations; others, on only a few hundred. The insert gives a rough indication of the average variability; it was calculated as follows. Since each individual time is, by assumption, exponential and independent, the sum, and so the mean, of the latencies obtained from a single exponential distribution has a gamma distribution. Because the ratio of the mean to the SD of a gamma distribution is the square root of the number of observations, we 

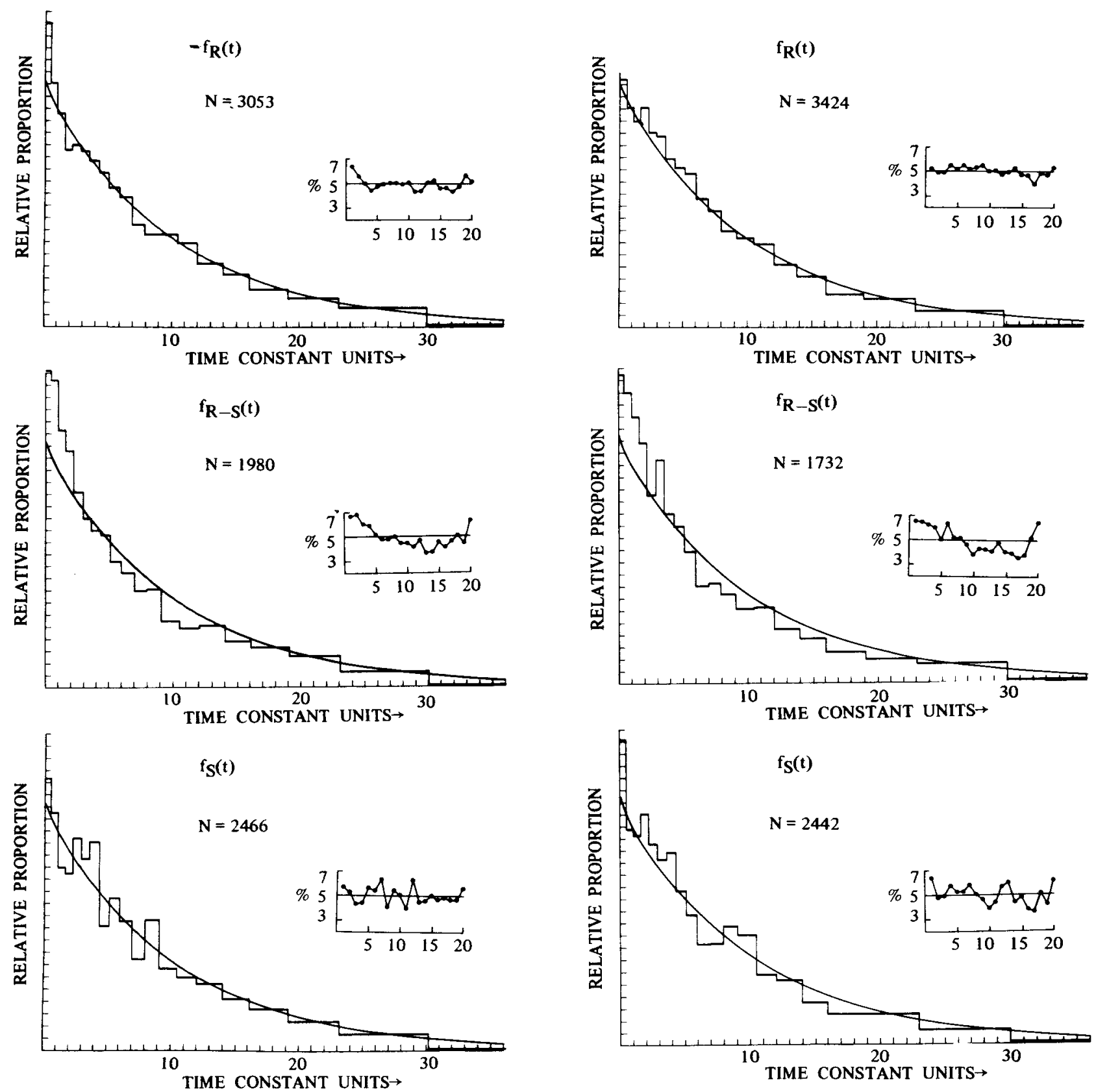

Fig. 6. Observed distributions of tail latencies for the three basic distributions in the experiment in which the signal level was varied $(\tau=1 / 2 \mathrm{sec})$. For each condition of the experiment, the expected and obtained percentage of latencies were determined for 20 equiprobable intervals of the exponential tails. The height of each rectangle in the histogram is such that the area is proportioned to the percentage of response falling in that interval. The inset in the panel is simply the obtained percentage for each of the $\mathbf{2 0}$ intervals. Were the data perfect, the height of each rectangle should fall along the exponential line and the insert should show a horizontal line at the value of $5 \%$. The graphs on the left are for 01 , those on the right, 02.

can easily estimate the SD. For example, if we have a mean of $2 \mathrm{sec}$ and 100 observations, then the $\mathrm{SD}$ in the estimate of the mean is about $0.2 \mathrm{sec}$; with 900 observations it is about 0.067 sec. Thus, the SD along the $x$ and $y$ axes of Fig. 8 can be estimated for each point and averaged over all of them. The insert displays that average.

We can also make the following test. First, as just described, estimate the variances of the $f_{R}$ and $f_{S}$ frequency distributions.
The difference between the two means divided by the sum of the variances should then be normally distributed with zero mean and unit variance. For $\mathrm{O} 1$ we found a mean of 0.3 (a positive difference occurs when the mean of $f_{R}$ is greater than that of $f_{S}$ ) and a SD of 1.22; for $O 2$ the mean was -1.5 and the SD was 2.5 . Thus, we conclude that some departure from perfect equality exists, especially for $\mathrm{O} 2$, but that it does not seem particularly serious. 

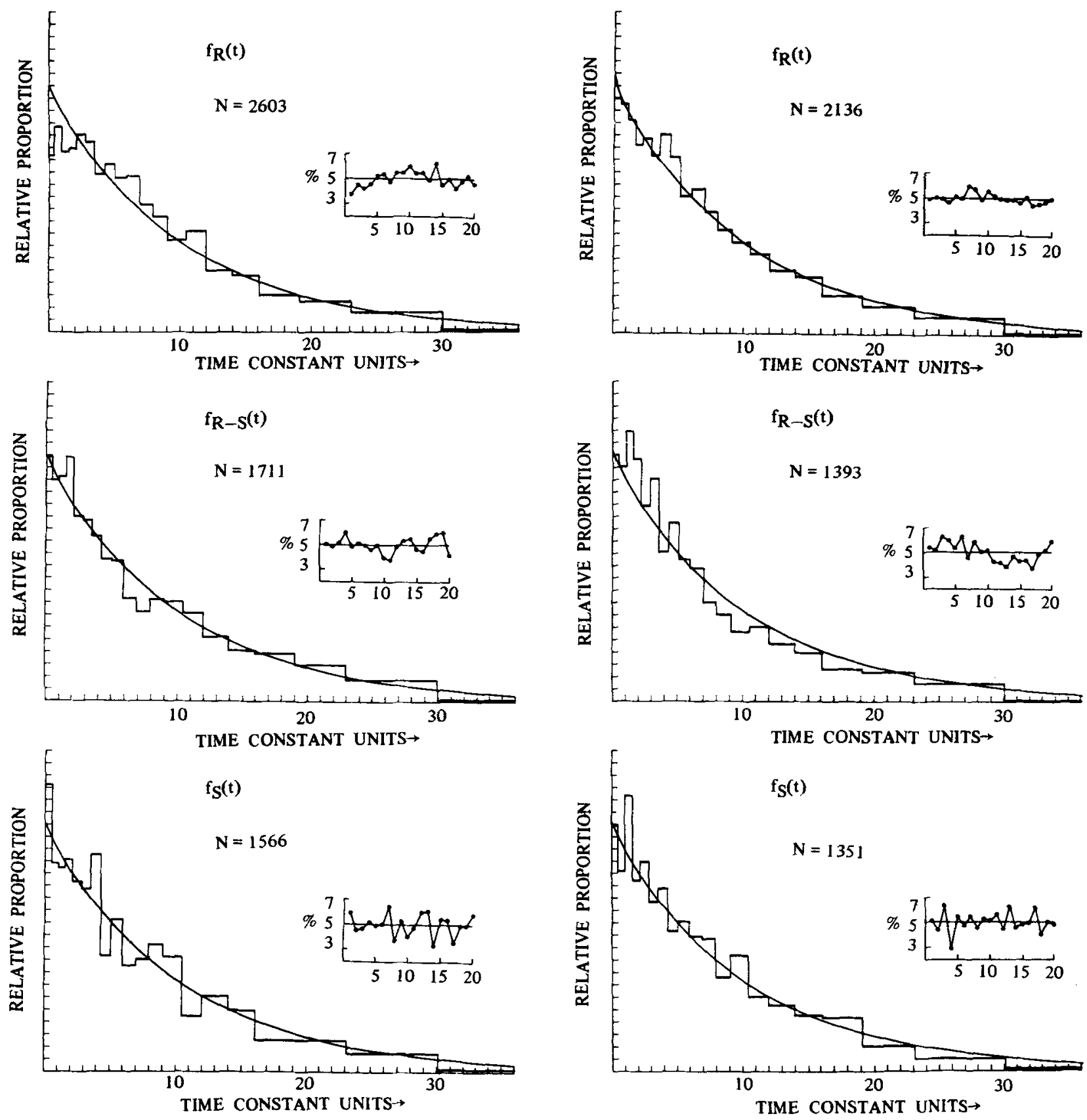

Fig. 7. The same as Fig. 6 except that the O's criteria were varied at a constant, intermediate signal level.

Do criteria changes affect only $b$ ? The parameter b $\mu$ is simply estimated by the exponential parameter of the $f_{R}-S$ distributions. We estimate bv by averaging the estimates of the exponential parameters for $f_{R}$ and $f_{S}$ and then subtracting $\lambda$. Call this average estimate $\overline{b u}$.

One of the simplest assumptions of the theory is that changes in an $O$ 's response criterion can be represented by changing the value $b$ of the response bias parameter. Thus, if we hold the physical conditions constant and induce the $O$ to vary his criterion, the ratio $b \mu / \bar{b} v$ should be independent of the criterion. These estimates, as a function of our three criterion regions, are shown in Table 1 along with range estimates given by $(\mathrm{b} \mu-\sigma) /(\mathrm{b} v+\sigma)$ and $(\mathrm{b} \mu+\sigma) /(\mathrm{b} v-\sigma)$. Both Os exhibit a definite decline in the ratio; however, there is plenty of overlap in the estimated ranges to include a constant value. Data from more Os will be needed before a definite conclusion can be drawn, but at present the hypothesis appears suspect. It looks as if there must be a sensory criterion that affects $\nu$ and $\mu$ somewhat differently. Such a theory should be developed.

Dependence of $b \mu / \overline{b v}$ on signal-to-noise ratio. Had we shown that $b \mu / b v$ was independent of $b$, then the dependence of this ratio on signal-to-noise ratio would be a function of considerable importance. As it is, we fear that the function shown in Fig. 9 would have been somewhat different had it been obtained for a different criterion. It is evident that the value of $b \mu / \overline{b v}$ is about 1 for very low signal-to-noise ratios, as it should be, and that it 


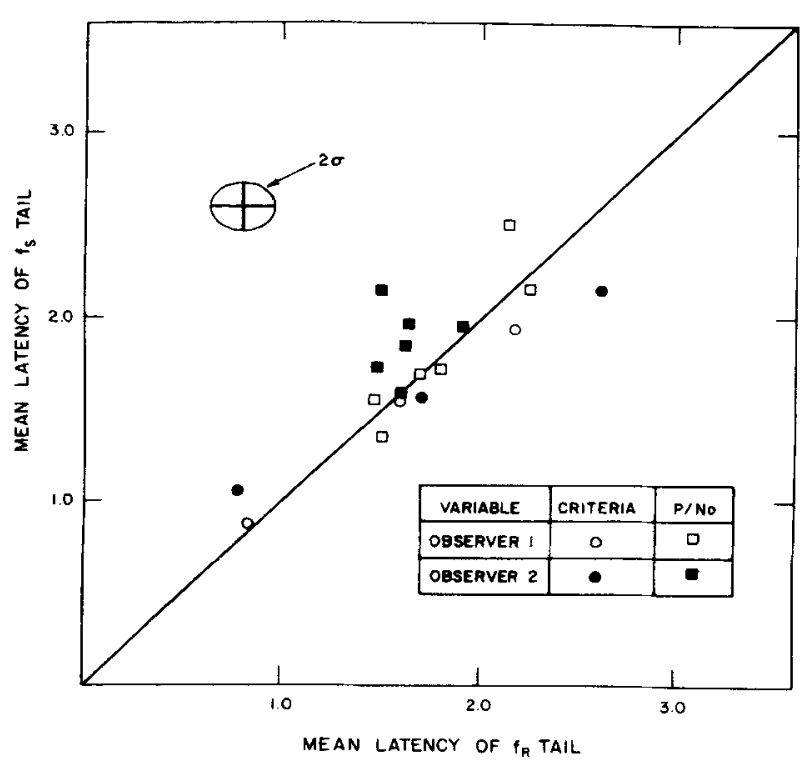

Fig. 8. Comparison of the mean latency of the tail $(\tau=1 / 2 \mathrm{sec})$ of the stimulus-wait and false-alarm distributions. According to the theory, the exponential parameters of each of these distributions are the same, thus the points should fall along the major diagonal.

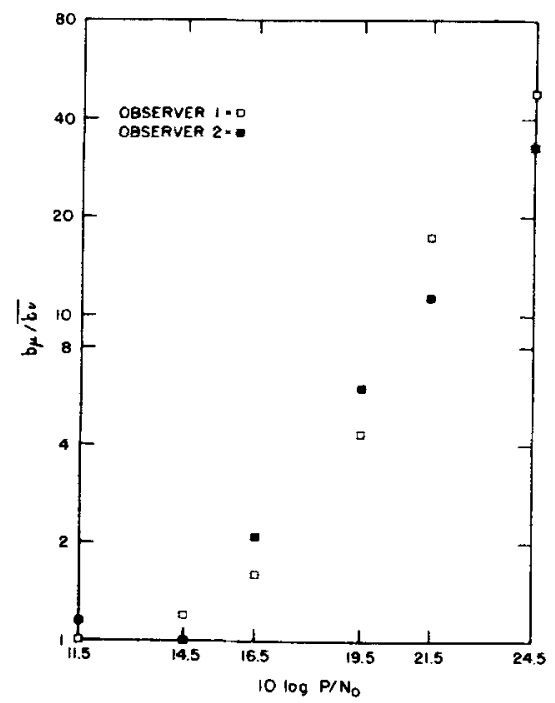

Fig. 9. The ratio of the two Poisson parameters, $b \mu / \overline{b v}$ vs the ratio of signal power, $P$, to noise density, $N_{0}$, in decibels. According to the theory, as the signal-to-noise ratio increases, the ratio of the two Poisson parameters should increase.

increases monotonically with that ratio; moreover, the functions for the two Os seem similar in shape, but slightly displaced. Notice that for $b \mu / \overline{b v}>1$, the $\log (b \mu / \overline{b v})$ is approximately linear with dB.

As these functions are analogous to psychometric functions measured by more conventional detection procedures, it is interesting to compare them with other measures of sensitivity. Since our signal is of unlimited duration, the data most directly comparable are those of Fletcher (1940) and of Hawkins and
Table 1

Estimates of $b \mu / b \nu$ for Different Response Criteria and of Its Range, Using $(b \mu-\sigma) /(b \nu+\sigma)$ and $(b \mu+\sigma) /(b \nu-\sigma)$ for the Upper and Lower Limits

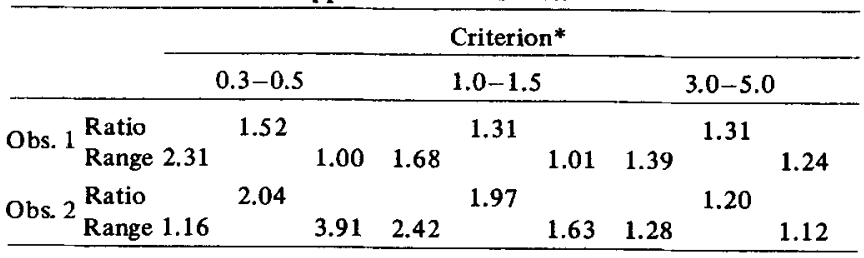

* Number of false alarms/Number of signals presented

Stevens (1950) on the threshold of a sinusoidal signal in white noise. In both cases the signal was on continuously and the $O$ adjusted its intensity until he could just detect it in noise. At $1,000 \mathrm{~Hz}$, the threshold $\mathrm{P} / \mathrm{N}_{0}$ level was from 16 to $18 \mathrm{~dB}$. According to Fig. 9, this corresponds to a ratio of about 2 in the Poisson parameters, which means that in any small interval of time, it is about twice as likely for the signal to initiate a response as for the noise to do so. This is an eminently reasonable definition of the threshold.

Mean observed reaction time. According to the model used in this analysis, the mean reaction time, as computed from the $f_{R-S}$ distribution

$$
\bar{f}_{R-S}=\int_{0}^{\infty} \mathrm{tf}_{R-S}(t) d t
$$

depends only on two statistics of the reaction density $r(t)$, namely, the mean reaction latency

$$
\bar{r}=\int_{0}^{\infty} r(t) d t
$$

and

$$
R_{-\lambda}=\int_{0}^{\infty} e^{-\lambda t} r(t) d t
$$

(see Eq. 38, Appendix 4). It is therefore of interest to try to estimate the mean reaction latency $\overline{\mathbf{r}}$ from the data collected in the various experimental conditions. Starting with Eq. 38, we can write

where

$$
y_{i}=\left(1-R_{-\lambda}\right) x_{i}+\bar{r}
$$

$$
y_{i}=\frac{\bar{f}_{R}-s, i-1 / b \mu_{i}}{1+b v_{i} / \lambda}
$$

and

$$
\mathbf{x}_{\mathrm{i}}=-\frac{\left(\overline{\mathbf{f}}_{\mathrm{R}-\mathrm{s}, \mathrm{i}}+1 / \lambda\right) \mathrm{b} v_{\mathrm{i}} / \lambda}{1+\mathrm{b} v_{\mathrm{i}} / \lambda}
$$

Thus, for the three criterion levels and six signal levels we can estimate $x_{i}$ and $y_{i}$. The scatter graph of these two parameters is shown in Fig. 10. A least squares fit to the points yields an estimate of $\bar{r}$ and $R_{-} \lambda$. The values of $\bar{r}$ so estimated are $292 \mathrm{msec}$ and $288 \mathrm{msec}$ for the Os. The two Os were also run in a typical reaction experiment with a very large signal intensity, namely, $50 \mathrm{~dB}$ above the noise power density. The same value of $\lambda$ was used so that the uncertainty about the starting time of the signal was the same. The mean reaction time was $251 \mathrm{msec}$ for $\mathrm{O} 1$ and $223 \mathrm{msec}$ for $\mathrm{O}$. The false-alarm rates were very low-less than $2 \%$.

Although the estimated values of $\vec{r}$ seem rather large, the 


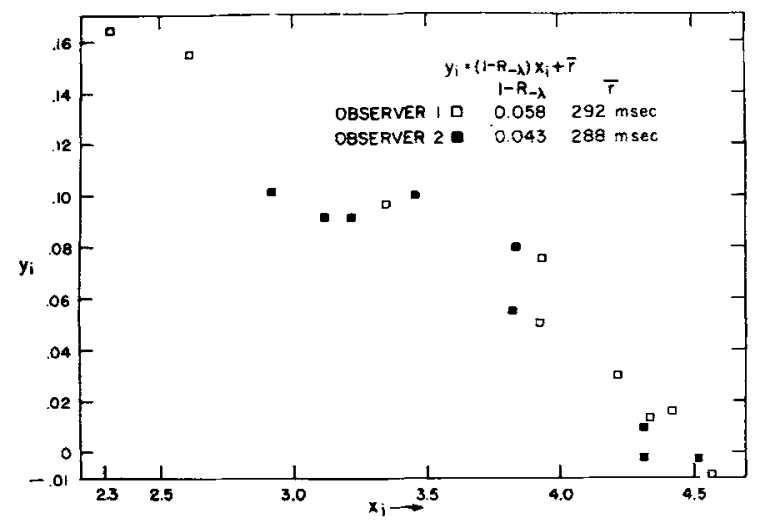

Fig. 10. Estimate of the (unobservable) mean reaction latency, $\overline{\mathbf{r}}$. For each of the nine experimental conditions, we estimated the various parameters and calculated a value of $x_{i}$ and $y_{i}$ according to Eq. 38 (also see text). A least squares fit of a straight line to each $O$ 's data yields estimates of $\bar{r}$ (the $y$ intercept) and of $1-\mathbf{R}_{-\lambda}$ (the slope).

consistency of these estimates over quite different conditions, and the correlation of these estimates with means measured under very strong signal-to-noise ratios, suggests that this aspect of the model deserves further exploration.

\section{APPENDIX 1}

\section{RESPONSE SUPPRESSION MODEL}

Assume that each input initiates an independent reaction latency, $r(t)$, that $r(t)$ is a continuous density equal to 0 for $t \geqslant \tau$, and that the first response to occur terminates all other incompleted reactions. Suppose that the inputs to the response process arrive according to a Poisson schedule with intensity $\alpha$ and that no reaction is under way at time 0 . Let $\mathrm{g}_{\alpha}$ denote the density and $G_{\alpha}$ the distribution of times to the next response, and let $R$ denote the distribution corresponding to density $r$. If the response occurs at time $t$, then we know that there was a first input at $x, 0 \leqslant x \leqslant t$, at which point a race began between that reaction and all of those reactions initiated by inputs arriving in the interval $(x, t)$. If we think of $x$ as a new origin, then the race is between one random variable with distribution $R$ and another with distribution $G_{\alpha}$, and the race is completed at time $t-x$. It is well known that the density of the smallest of $n$ independent random variables with distribution functions $H_{i}, i=1, \cdots, n$, is given by

$$
-\frac{d}{d t} \prod_{i=1}^{n}\left[1-H_{i}(t)\right]
$$

so we see that

$$
\begin{aligned}
G_{\alpha}^{\prime}(t)=g_{\alpha}(t) & =-\int_{0}^{t} \alpha e^{-\alpha x} \frac{d}{d x}\left\{[1-R(t-x)]\left[1-G_{\alpha}(t-x)\right]\right\} d x \\
& =-\alpha e^{-\alpha t} \int_{0}^{t} e^{\alpha y} \frac{d}{d y}\left\{[1-R(y)]\left[1-G_{\alpha}(y)\right]\right\} d y
\end{aligned}
$$

Differentiate Eq. 1,

$$
\begin{aligned}
G_{\alpha}^{\prime \prime}(t) & =-\alpha \frac{d}{d t}\left\{[1-R(t)]\left[1-G_{\alpha}(t)\right]\right\}-\alpha G_{\alpha}^{\prime}(t) \\
& =\alpha R^{\prime}(t)-\alpha \frac{d}{d t}\left[R(t) G_{\alpha}(t)\right]
\end{aligned}
$$

Integrate this last equation and note that, since $r$ is continuous, $R(0)=0$, and by Eq. 1, $G^{\prime}(0)=0$, so

$$
\alpha R(t)=G_{\alpha}^{\prime}(t) /\left[1-G_{\alpha}(t)\right],
$$

which is the hazard function of $g_{\alpha}$ So, as is well known,

$$
1-G_{\alpha}(t)=\exp \left[-\alpha \int_{0}^{t} R(x) d x\right] .
$$

Since $r(t)=0$ for $t \geqslant \tau$, integration by parts shows that

$$
\int_{0}^{t} R(x) d x=t-\bar{r}, \quad t \geqslant \tau,
$$

where $\bar{r}$ is the mean reaction latency, i.e.,

$$
\bar{r}=\int_{0}^{\tau} \operatorname{tr}(t) d t
$$

By differentiating Eq. 2 and using Eq. 3, we obtain

$$
g_{\alpha}(t)=\alpha e^{-\alpha t_{e} \alpha \bar{\tau}}, \quad t \geqslant \tau
$$

Let the densities $\mathbf{f}_{\mathbf{i}}$ be defined as in the section on Alternative Models. Let $b \eta=b v+\lambda b q$, then by Eq. 5 ,

$$
\begin{aligned}
f_{1}(t) & =g_{b} \eta^{(t)} \\
& =\left[e^{b \eta \bar{x}}\right] b \eta e^{-b \eta t}, \quad t \geqslant \tau .
\end{aligned}
$$

Integrating Eq. 7, and solving,

$$
\bar{r}=\tau+\frac{1}{b \eta} \ln \left[\int_{\tau}^{\infty} f_{1}(t) d t\right] .
$$

The IRT density conditional on no intervening signal is

$$
\begin{aligned}
f_{2}(t) & =\frac{g_{b v}(t) e^{-\lambda t}}{R_{b v,-\lambda}} \\
& =\left[\frac{b v e^{b v \bar{r}}}{(b v+\lambda) R_{b v,-\lambda}}\right](b v+\lambda) e^{-(b v+\lambda) t}, t \geqslant \tau,
\end{aligned}
$$

where, from Eq. 2,

$$
\begin{aligned}
\mathbf{R}_{\mathrm{b} v,-\lambda} & =\int_{0}^{\infty} \mathrm{g}_{\mathrm{b} v}(\mathrm{t}) \mathrm{e}^{-\lambda \mathrm{t}} \mathrm{dt} \\
& =\int_{0}^{\infty} \mathrm{b} u \mathrm{R}(\mathrm{t}) \exp \left[-\mathrm{b} v \int_{0}^{\mathrm{t}} \mathrm{R}(\mathrm{x}) \mathrm{dx}-\lambda \mathrm{t}\right] \mathrm{dt} .
\end{aligned}
$$

For the two SRT densities conditional on no observable event $\tau \sec$ prior to the signal, we locate the signal's occurrence at time $\tau$ and the response at time $\tau+\mathrm{t}$. Since the conditioning is over a fixed interval, it factors from both numerator and denominator. It does, however, imply that no reaction was in progress at time 0 , and so we have a race between noise-induced reactions throughout $(0, \tau+t)$, signal-induced reactions in the interval $(\tau, \tau+t)$, and, with probability bq, a reaction latency of $t \sec$. Therefore, by Eq. 2 ,

$$
\begin{aligned}
f_{3}(t) & =\frac{-\frac{d}{d t}\left\{[1-b q R(t)]\left[1-G_{b v}(\tau+t)\right]\left[1-G_{\lambda b q}(t)\right]\right\}}{-\int_{0}^{\infty} \frac{d}{d t}\left\{[1-b q R(t)]\left[1-G_{b v}(\tau+t)\right]\left[1-G_{\lambda b q}(t)\right]\right\} d t} \\
& =\frac{-\frac{d}{d t}\left\{[1-b q R(t)] \exp \left[-b v \int_{0}^{\tau+t} R(x) d x-\lambda b q \int_{0}^{t} R(x) d x\right]\right\}}{e^{-b v(\tau-\bar{r})}}
\end{aligned}
$$




$$
=\left[(1-b q) e^{\lambda b q \overline{\mathbf{r}}}\right] b \eta e^{-b \eta t}, \quad t \geqslant \tau .
$$

The analysis of $f_{4}$ is similar except that the condition of no signal in $(\tau, \tau+t)$ means that the term $1-G \lambda_{b q}(t)$ is dropped from the derivative and the factor $e^{-\lambda t}$ is added. Thus, using Eqs. 2 and 3 and in tegrating by parts twice,

$$
\begin{aligned}
-\int_{0}^{\infty} \mathrm{e}^{-\lambda \mathrm{t}} & \frac{\mathrm{d}}{\mathrm{dt}}\left\{[1-\mathrm{bqR}(\mathrm{t})]\left[1-\mathrm{G}_{\mathrm{b} v}(\tau+\mathrm{t})\right]\right\} \\
= & -\left.\mathrm{e}^{-\lambda \mathrm{t}}[1-\mathrm{bqR}(\mathrm{t})]\left[1-\mathrm{G}_{\mathrm{b} v}(\tau+\mathrm{t})\right]\right|_{0} ^{\infty} \\
& -\int_{0}^{\infty} \lambda \mathrm{e}^{-\lambda \mathrm{t}}[1-\mathrm{bqR}(\mathrm{t})] \exp \left[-\mathrm{b} v \int_{0}^{\tau} \mathrm{R}(\mathrm{x}) \mathrm{dx}-\mathrm{b} v \mathrm{t}\right] \mathrm{dt} \\
= & \exp \left[-\mathrm{b} v \int_{0}^{\tau} \mathrm{R}(\mathrm{x}) \mathrm{dx}\right]\left\{1+\frac{\lambda}{(\mathrm{b} v+\lambda)}[1-\mathrm{bqR}(\mathrm{t})] \mathrm{e}^{-\left.(\mathrm{b} v+\lambda) \mathrm{t}\right|_{0} ^{\infty}}\right. \\
& \left.+\frac{\lambda}{(\mathrm{b} v+\lambda)} \int_{0}^{\infty} \mathrm{bqr}(\mathrm{t}) \mathrm{e}^{-(\mathrm{b} v+\lambda) \mathrm{t}} \mathrm{dt}\right\} \\
= & \mathrm{e}^{-\mathrm{b} v(\tau-\overrightarrow{\mathrm{r}})}\left[1+\lambda \frac{\mathrm{bq}}{\mathrm{b} v} \mathrm{R}-(\mathrm{b} v+\lambda)\right] \frac{\mathrm{b} v}{(\mathrm{~b} v+\lambda)},
\end{aligned}
$$

where, as in the lock-out model,

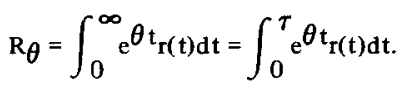

Thus,

$$
\begin{aligned}
& f_{4}(t)=\frac{-e^{-\lambda t} \frac{d}{d t}\left\{[1-b q R(t)] \exp \left[-b v \int_{0}^{\tau+t} R(x) d x\right]\right\}}{e^{-b v(\tau-\bar{r})}\left[1+\lambda \frac{b q}{b v} R_{-(b v+\lambda)}\right] b v /(b v+\lambda)} \\
& =\left[\frac{1-b q}{1+\lambda \frac{b q}{b v} R_{-(b v+\lambda)}}\right](b v+\lambda) e^{-(b v+\lambda) t}, t \geqslant \tau
\end{aligned}
$$

Finally, suppose that the previous response is located at the origin, the first signal at $x$, and the next response at $x+t$, then by the same sort of argument

$f_{5}(t)$

$$
=\frac{-\int_{0}^{\infty} \lambda e^{-\lambda x} \frac{d}{d t}\left\{[1-b q R(t)]\left[1-G_{b v}(x+t)\right]\left[1-G_{\lambda b q}(t)\right]\right\} d x}{-\int_{0}^{\infty} \int_{0}^{\infty} \lambda e^{-\lambda x} \frac{d}{d t}\left\{[1-b q R(t)]\left[1-G_{b v}(x+t)\right]\left[1-G_{\lambda b q}(t)\right]\right\} d x d t}
$$

The denominator is evaluated by interchanging the order of integration, which is permissible since the functions are continuous and the region of integration is a rectangle, and then integrating by parts. The result is $(1-b q)\left(1-R_{b} v,-\lambda\right)$, where $R_{b} v,-\lambda$ is defined by Eq. 11 . For $t \geqslant \tau$, we substitute from Eqs. 2 and 3 in the numerator, differentiate, and then integrate the result to obtain

$$
f_{5}(t)=\left[\frac{e^{b \eta \bar{r}}}{(b v+\lambda)\left(1-R_{b v,-\lambda)}\right.}\right] b \eta e^{-b \eta t}, t \geqslant \tau .
$$

\section{APPENDIX 2 \\ QUEUE MODEL}

Assume that inputs arrive according to a Poisson schedule with intensity $\mathrm{b} n$, that there is a first-in-first-out memory, and that the bounded reaction latency has density r. If $\rho=b \eta \bar{r}<1$, where $\overline{\mathbf{r}}$ is the mean reaction latency
(Eq. 4), it is known that $\rho$ is the asymptotic probability that the memory is non-empty when a response is made (Cox \& Smith, 1961, p. 53).

For $f_{1}, f_{2}$, and $f_{5}$ we locate the previous response at the origin. With probability $\rho$ there is an input in memory and with probability $1-\rho$ there is not. In the former case, the density of $t$ is the reaction latency density $r(t)$; whereas, in the latter, it is simply $f_{1}$ of the lock-out model. So we may write immediately

$$
\begin{aligned}
& \mathrm{f}_{1}(\mathrm{t})=\rho \mathrm{r}(\mathrm{t})+(1-\rho) \mathrm{b} \eta \mathrm{e}^{-\mathrm{b} \eta \mathrm{t}} \int_{0}^{\mathrm{t}} \mathrm{e}^{\mathrm{b} \eta \mathrm{y}_{\mathrm{r}}(\mathrm{y}) d \mathrm{y}} \\
& =\left[(1-\rho) \mathrm{R}_{\mathrm{b} \eta}\right] \mathrm{b} \eta \mathrm{e}^{-\mathrm{b} \eta \mathrm{t},} \mathrm{t} \geqslant \tau .
\end{aligned}
$$

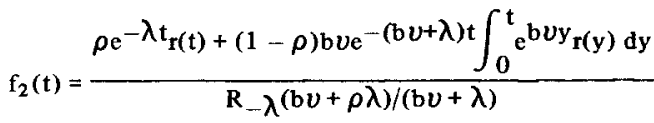

$$
\begin{aligned}
& =\left[\frac{(1-\rho) \mathrm{b} v}{(\mathrm{~b} v+\rho \lambda)} \frac{\mathrm{R}_{\mathrm{b} v}}{\mathrm{R}_{-\lambda}}\right](\mathrm{b} v+\lambda) \mathrm{e}^{-(\mathrm{b} v+\lambda) \mathrm{t}, \quad \mathrm{t} \geqslant \tau .}
\end{aligned}
$$

To calculate $f_{5}$ we must proceed in greater detail since we have not previously calculated it for the lock-out model. The numerator is

$$
\begin{aligned}
& h(t)=\int_{0}^{\infty} \lambda e^{-\lambda x}\left\{\rho \mathrm{r}(\mathrm{x}+\mathrm{t})+(1-\rho)\left[\int_{0}^{\mathrm{x}} \mathrm{b}^{-\mathrm{b} v \mathrm{y}_{\mathrm{r}(\mathrm{t}+\mathrm{x}-\mathrm{y}) \mathrm{dy}}}\right.\right.
\end{aligned}
$$

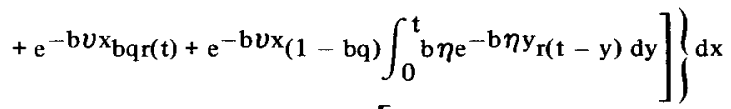

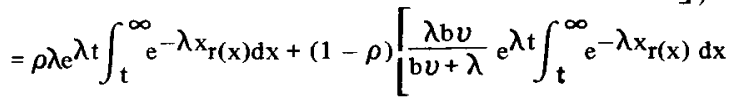

$$
\begin{aligned}
& \left.+\frac{\lambda \mathrm{bq}}{\mathrm{b} v+\lambda} \mathrm{r}(\mathrm{t})+\frac{\lambda(1-\mathrm{bq})}{\mathrm{b} v+\lambda} \mathrm{b} \eta \mathrm{e}^{-\mathrm{b} \eta \mathrm{t}} \int_{0}^{\mathrm{t}} \mathrm{e}^{\mathrm{b} \eta \mathrm{x}_{\mathrm{T}}(\mathrm{x}) \mathrm{dx}}\right] \text {. }
\end{aligned}
$$$$
\int_{0}^{\infty} h(t) d t
$$

$$
=\rho\left(\mathrm{R}_{-\lambda}+1\right)+(1-\rho)\left[\frac{\mathrm{b} v}{\mathrm{~b} v+\lambda}\left(\mathrm{R}_{-} \lambda+1\right)+\frac{\lambda \mathrm{bq}}{\mathrm{b} v+\lambda}+\frac{\lambda(1-\mathrm{bq})}{\mathrm{b} v+\lambda}\right] .
$$

Thus,

$f_{5}(t)=(1-\rho)\left[\frac{(1-b q) R_{b}}{R_{-\lambda}\left(\rho+\frac{b v}{\lambda}\right)+\frac{b v}{\lambda}+1}\right] b \eta e^{-b \eta t}, \quad t \geqslant \tau$

For the two SRT densities conditional on no signal or response during the $\tau$ sec before the signal in question, $f_{3}$ and $f_{4}$, we know from the boundedness of the reaction latency that there was no reaction in progress at the beginning of the conditioning interval. Thus, the predictions are exactly the same as for the lock-out model.

\section{APPENDIX 3 \\ LOCK-OUT MODEL WITH SIGNALS OF FINITE DURATION}

All assumptions of this model are the same as in the original lock-out model, except that the signal is represented as a Poisson process with parameter $b \mu-b v$ (above the noise level $b v$ ) of fixed duration $\delta$. The interval from the end of one signal to the beginning of the next is exponential with time constant $\lambda$. Considering only the signals and ignoring the noise, let 0 denote a time when no reaction is in progress and let $t$ be the time of the next signal-induced detection. We wish to calculate the density $g(t)$ of such detection times assuming no noise-induced detection. Observe that any signal that begins before $t-\delta$ cannot possibly have 
fathered the detect state. So, if the first signal occurs at $x, 0 \leqslant x \leqslant t-\delta$, which it does with probability $\lambda e^{-\lambda x}$ and if there is no detection caused by the signal that has probability $\mathrm{e}^{-(\mathrm{b} \mu-\mathrm{b} v) \delta}$, then the process begins anew with $t-\delta-x$ sec remaining. Alternatively, if the first signal begins later than $\mathbf{t}-\delta$, then it must terminate in a detection. Thus,

$$
\begin{aligned}
g(t)= & \int_{0}^{t-\delta} \lambda e^{-\lambda x_{e}-(b \mu-b v) \delta} g(t-\delta-x) d x \\
& +\int_{0}^{\delta} \lambda e^{-\lambda(t-\delta+x)}(b \mu-b v) e^{-(b \mu-b v)(\delta-x)} d x \\
= & \lambda e^{-\lambda(t-\delta)} e^{-(b \mu-b v) \delta} \int_{0}^{t-\delta} e^{\lambda y_{g}(y) d y} \\
& +e^{-\lambda t} A(\lambda, b \mu-b v, \delta) .
\end{aligned}
$$

Multiply by $\mathrm{e}^{\lambda t}$, differentiate, and then multiply by $\mathrm{e}^{-\lambda t}$ :

$$
g^{\prime}(t)+\lambda g(t)=\lambda e^{-(b \mu-b v)} \delta_{g(t-\delta)} .
$$

Assuming that $\delta$ is so small-0.1 or less-that terms $\delta^{2}$ and higher are negligible in Taylor's expansion of $\mathrm{g}(\mathrm{t}-\delta)$, we readily obtain

$$
g(t)=\alpha \lambda e^{-\alpha \lambda t}
$$

where

$$
\alpha=\frac{1-\mathrm{e}^{-(\mathrm{b} \mu-\mathrm{b} v) \delta}}{1+\lambda \delta \mathrm{e}^{-(\mathrm{b} \mu-\mathrm{b} v) \delta}}
$$

The IRT density then is a race between a signal-induced output, with the density given by Eq. 24, and a noise-induced output, with density bue $e^{-b v t}$, to which the reaction latency is appended, so

$$
\begin{aligned}
f_{1}(t) & =\int_{0}^{t}-\frac{d}{d x}\left\{[1-G(x)] e^{-b v x}\right\} r(t-x) d x \\
& =(b v+\alpha \lambda) e^{-(b v+\alpha \lambda)} \int_{0}^{t} e^{(b v+\alpha \lambda) y_{r(y) d y}}
\end{aligned}
$$

which is exactly the same equation as in the original idealized lock-out model if we set $b \eta=b v+\alpha \lambda$. Thus, the estimate of bq in that model and of $\alpha$ in this one are the same. So, from Eq. 25 we obtain

$$
\begin{aligned}
\frac{\mathrm{b} \mu}{\mathrm{b} v} & =1+\frac{1}{\delta \mathrm{b} v} \ln \left(\frac{1+\alpha \lambda \delta}{1-\alpha}\right) \\
& \approx 1+\frac{\alpha}{\delta \mathrm{b} v}+\frac{\alpha \lambda}{\mathrm{b} v}, \text { for } \alpha \text { small, and } \lambda \delta \text { near zero. }
\end{aligned}
$$

Since $f_{2}$ is conditional on no intervening signal, it is unchanged.

In the calculations for $f_{3}, f_{4}$, and $f_{5}$, the numerator differs depending on whether $t \lessgtr \delta$. We set it up explicitly for $f_{3}$ and simply remark that $f_{4}$ and $f_{5}$ are analogous. For $t \leqslant \delta$, we have

$$
\begin{aligned}
& \int_{0}^{\tau} b v e^{-b v x_{r}(\tau+t-x) d x} \\
& \quad+e^{-b v r} \int_{0}^{t}-\frac{d}{d x}\left[e^{-b v x_{e}-(b \mu-b v) x}\right] r(t-x) d x \\
& =b v e^{-b v(\tau+t)} \int_{t}^{t+\tau} e^{b v y} r(y) d y \\
& \quad+b \mu e^{-b v \tau_{e}-b \mu t} \int_{0}^{t} e^{b \mu y_{r}(y) d y}
\end{aligned}
$$

And for $t \geqslant \delta$, we have

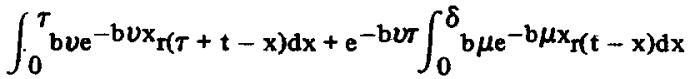

$$
\begin{aligned}
& +e^{-b v \tau_{e}-b \mu \delta} \int_{0}^{t-\delta}-\frac{d}{d x}\left\{[1-G(x)] e^{-b v x}\right\} r(t-\delta-x) d x
\end{aligned}
$$

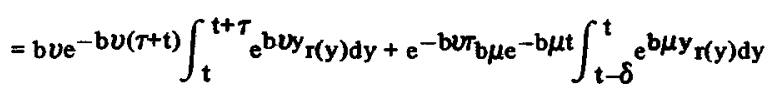

$$
\begin{aligned}
& +e^{-b v \pi_{e}-b \mu \delta_{b} e^{-b} \eta(t-\delta)} \int_{0}^{t-\delta} e^{b \eta y_{r(y)}} d y
\end{aligned}
$$

Integrating the numerator from 0 to $\infty$ involves integrating the first expression from 0 to $\delta$ and the second from $\delta$ to $\infty$. Integrating by parts

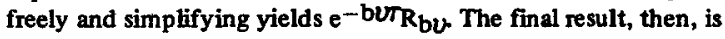

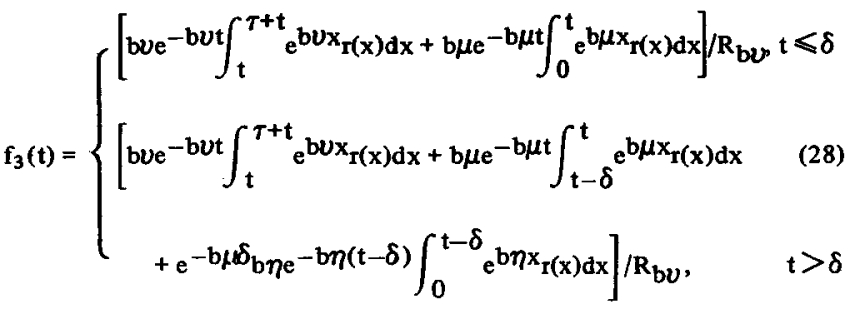

$$
\begin{aligned}
& f_{3}(t)=\left[e^{-(b u-b n) \delta} \frac{R_{b \eta}}{R_{b v}}\right] b \eta e^{-b \eta t}, t \geqslant \tau+\delta .
\end{aligned}
$$

Similarly, let

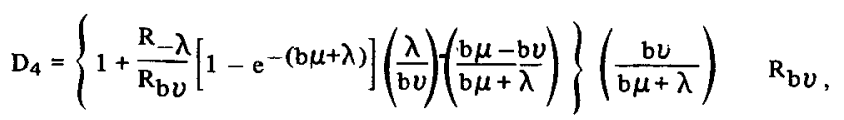

then

$\mathbf{f}_{4}(\mathrm{t})$

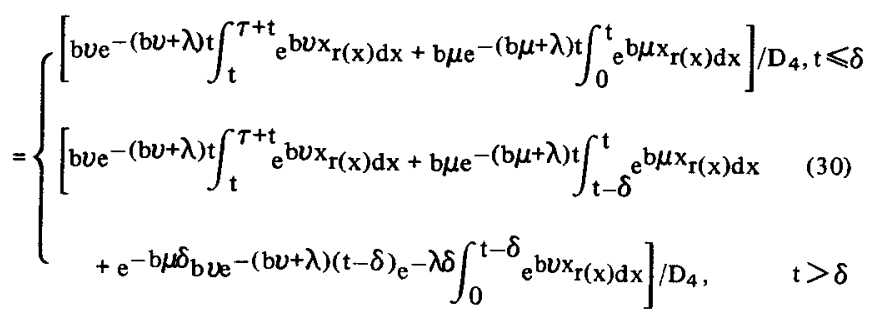

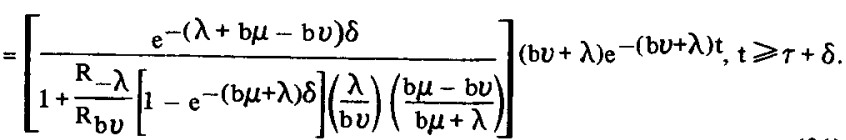


then

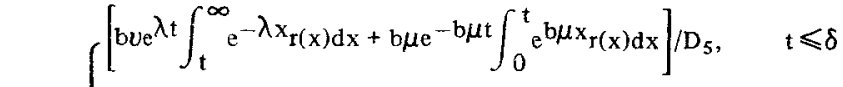

$$
\begin{aligned}
& f_{5}(t)=\left\{\left[b v e^{\lambda t} \int_{t}^{\infty} e^{-\lambda x_{r}(x) d x+b \mu e^{-b \mu t}} \int_{t-\delta}^{t} e^{b \mu x_{r}(x) d x}\right.\right. \\
& \left.b \eta \mathrm{e}^{-\mathrm{b} \mu \delta_{\mathrm{e}}-\mathrm{b} \eta(\mathrm{t}-\delta)} \int_{0}^{\mathrm{t}-\delta} \mathrm{e}^{\mathrm{b} \eta \mathrm{x}_{\mathrm{r}}(\mathrm{x}) \mathrm{dx}}\right] / \mathrm{D}_{5} \\
& f_{5}(t)=\left[\frac{R_{b} \eta^{-(b \mu-b \eta) \delta}}{1+\frac{b v}{\lambda}\left(R_{-}+1\right)}\right] b \eta e^{-b \eta t}, t \geqslant \tau+\delta
\end{aligned}
$$

Observe that when $\lambda, \delta$, and bv are all in the neighborhood of 0.1 , as they are in our experiments, then factors of the form $e^{b u \delta}, e^{b} \eta \delta$, and $e^{-\lambda \delta}$ are all virtually 1 , so all of the exponential factors can be replaced by $\mathrm{e}^{-(\mathrm{b} \mu-\mathrm{b} v) \delta}$ which, by Eq. 25 , is approximately $1-\alpha=1-\mathrm{bq}$. Therefore, Eqs. 29, 31, and 33 are approximately the same as for the original lock-out model.

\section{APPENDIX 4}

\section{REACTION TIME MODEL}

The model was described in detail in the body of the paper. Locate the beginning of the trial at the origin. One mechanism, to be described, results in an unobservable output that in turn initiates a bounded reaction latency with density $I$ and bound $\tau$. The overall response time random variable is denoted by $R$. The output is controlled by a Poisson process that has intensity $b u$ from 0 until the onset of the signal and intensity $b \mu \geqslant b v$ thereafter. The time from 0 to the signal onset is an exponentially distributed random variable $S$ with time constant $\lambda$. We derive three densities.

The first is the false alarm density of times to the response when the response precedes the signal, i.e., the density that $R=t$ given that $S>R$. Since $S>R$, we know that a noise output occurred at some time $x$, $0 \leqslant x \leqslant t$, that the reaction latency was $t-x$, that no signal occurred in $(0, t)$. These events are, by assumption, statistically independent. Thus,

$$
\begin{aligned}
f_{R}(t) & =\operatorname{Pr}(R=t \mid S>R) \\
& =\frac{\operatorname{Pr}[(R=t) \cap(S>t)]}{\operatorname{Pr}(S>R)} \\
& =\frac{\int_{0}^{t} b v e^{-b v x_{r}(t-x) e^{-\lambda t} d x}}{\int_{0}^{\infty} \int_{0}^{t} b v e^{-b v x_{r}(t-x) e^{-\lambda t} d x d t}} \\
& =\left[(b v+\lambda) e^{-(b v+\lambda) t} \int_{0}^{t} e^{b v y_{r}(y) d y}\right] / R_{-\lambda} \\
& =\left[R_{b v} / R_{-\lambda}\right](b v+\lambda) e^{-(b v+\lambda) t}, t>\tau .
\end{aligned}
$$

The other two densities concern the case when $S \leqslant R$. Suppose that $S=x$ and $R-S=t$, and let $y$ be the time of the output that initiated the reaction. Thus, the reaction latency $x+t-y$. The density of $y$ divides into two parts depending on the relation of $y$ to $x$. If $y \leqslant x$, it is bve

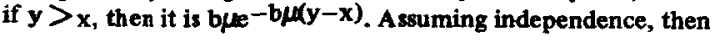

$$
\begin{aligned}
& \operatorname{Pr}[(R-S=t) \cap(t \geqslant 0) \cap(S=x)] \\
& =\lambda e^{-\lambda x}\left[\int_{0}^{x} b v e^{-b v y_{r}(t+x-y) d y}\right. \\
& \left.\quad+e^{-\underline{b} v x} \int_{x}^{x+t} b \mu e^{-b \mu(y-x)} r(t+x-y) d y\right] .
\end{aligned}
$$

Simplifying and integrating by parts, it is not difficult to show that the reaction time density is given by

$$
\begin{aligned}
f_{R-S}(t) & =\operatorname{Pr}(R-S=t \mid S \leqslant R) \\
& =\frac{\int_{0}^{\infty} \operatorname{Pr}[(R-S=t) \cap(t \geqslant 0) \cap(S=x)] d x}{\int_{0}^{\infty} \int_{0}^{\infty} \operatorname{Pr}[(R-S=t) \cap(t \geqslant 0) \cap(S=x)] d x d t} \\
& =\frac{\lambda b v e^{\lambda t} \int_{t}^{\infty} e^{-\lambda z_{r}(z) d z+\lambda b \mu e^{-b \mu t} \int_{0}^{t} e^{b \mu z_{r}(z) d z}}}{b v\left(1-R_{-}\right)+\lambda} \\
& =\left[\frac{R_{b} \mu}{1+\frac{b v}{\lambda}\left(1-R_{-\lambda}\right)}\right] b \mu e^{-b \mu t}, t \geqslant \tau .
\end{aligned}
$$

From Eq. 36, the mean reaction time may be computed by integrating by parts:

$$
\int_{0}^{\infty} \mathrm{tf}_{\mathrm{R}-\mathrm{S}}(\mathrm{t}) \mathrm{dt}=\frac{\overline{\mathrm{T}}(1+\mathrm{b} v / \lambda)+(1 / \mathrm{b} \mu)-\left(1-\mathrm{R}_{-\lambda}\right)(\mathrm{b} v / \lambda)(1 / \lambda)}{1+(\mathrm{b} v / \lambda)\left(1-\mathrm{R}_{-\lambda}\right)}
$$

Note that it is proportional to the mean reaction latency, $\overline{\mathbf{r}}$, and inversely proportional to signal strength, $b \mu$.

Next we examine the signal-wait density of times to the signal conditional on the signal preceding the response. The response will follow the signal either because no noise output occurs before the signal, which has probability $e^{-b v t}$, or because one does occur at $x \leqslant t$ and the response latency is sufficiently long $-t-x+y$, where $y \geqslant 0$. Thus,

$\operatorname{Pr}[(S=t) \cap(R>t)]=\lambda e^{-\lambda t}\left[e^{-b v t}+\int_{0}^{\infty} \int_{0}^{t} b v e^{-b v x_{r}(t-x+y) d x d y}\right]$.

From this it is routine to show that

$$
\begin{aligned}
f_{S}(t) & =\operatorname{Pr}(S=t \mid S \leqslant R) \\
& =\frac{\lambda(b v+\lambda)\left[e^{-(b v+\lambda) t} \int_{0}^{t} e^{b v z_{r}(z) d z+e^{-\lambda t} \int_{t}^{\infty} r(z) d z}\right]}{b v\left(1-R_{-\lambda}\right)+\lambda} \\
& =\left[\frac{R_{b v}}{1+\frac{b v}{\lambda}\left(1-R_{-\lambda}\right)}\right](b v+\lambda) e^{-(b v+\lambda) t}, t \geqslant \tau .
\end{aligned}
$$

\section{REFERENCES}

BROADBENT, D. E., \& GREGORY, M. Vigilance considered as a statistical decision. British Journal of Psychology, 1963, 54, 309-323.

COX, D. R., \& SMITH, W. L. Queues. London: Methuen; New York: Wiley, 1961 .

EGAN, J. P., GREENBERG, G. Z., \& SCHULMAN, A. I. Operating characteristics, signal detectability, and the method of free response. Joumal of the Acoustical Society of America, 1961, 33, 993-1007.

FLETCHER, H. Auditory patterns. Review of Modern Physics, 1940, 12, $47-65$.

GREEN, D. M., \& LUCE R. D. Detection of auditory signals presented at random times. Perception \& Psychophy sics, 1967, 2, 441-449.

HAWKINS, J. E., JR., \& STEVENS, S. S. The masking of pure tones and of speech by white noise. Journal of the Acoustical Society of America, 1950, 22, 6-13.

LUCAS, P. A. Human performance in low-signal-probability tasks. Journal of the Acoustical Society of America, 1967, 42, 158-178.

LUCE, R. D. A model for detection in temporally unstructured experiments with a Poisson distribution of signal presentations. Journal of Mathemratical Psychology, 1966, 3, 48-64.

WATSON, C. S., \& NICHOLS, T. L. Replication and revisions of Egan's method of free respense. (Abstract) Journal of the Acoustical Society of America, 1966, 36, 1247. 
NOTES

1. This research was supported by Grant GB7009 from the National Science Foundation. The experimentation was carried out on computer facilities supported by Grant NB07454 from the National Institutes of Health. We are deeply indebted to Miss Virginia L. Maier who prepared the programs needed to nu the experiments and for the programs used to analyze the data. David E. Rumelhart was kind enough to read and check some of the equations for us.

2. This work was carried out when Dr. Luce was affiliated with the University of Pennsylvania and was on leave as an Organization of
American States Professor at the Pontificia Universidade Católica do Rio de Janeiro.

3. Address: Psychology Department, University of California, San Diego, La Jolla, California 92037.

4. We use the convention of referring to observable temporal random variables as "times" and unobservable ones as "latencies."

5. The error arose in programming the prediction equation. The programmed equation was checked, but regrettably only at values where the correct and incorrect prediction were nearly the same. At other values of the arguments, the predictions are quite different. 